\title{
Dynamics of Hypernuclear Nonmesonic Weak Decay
}

\author{
Franjo Krmpotić ${ }^{*}$, Airton Deppman ${ }^{\dagger}$ and Claudio De Conti** \\ *Instituto de Física La Plata, CONICET, \\ Universidad Nacional de La Plata, 1900 La Plata, Argentina. \\ ${ }^{\dagger}$ Instituto de Física, Universidade de São Paulo, São Paulo, Brasil \\ ** Universidade Estadual Paulista, 18409-010, Itapeva, SP, Brazil
}

\begin{abstract}
Simple and coincidence spectra of the NM weak decay of light hypernuclei have been evaluated in a systematic way for the first time. We have only considered $1 \mathrm{~N}$ induced processes, neglecting entirely the events induced by $2 \mathrm{~N}$ emission, as well as the effects of the FSIt's. As the theoretical framework we have used the IPSM with three different parametrizations for the transition potential. The comparison with data strongly suggests that the soft $\pi+K$ exchange model could be a good starting point to describe the dynamics in the NM weak decays of $s$ - and $p$-shell hypernuclei.
\end{abstract}

Keywords: nonmesonic weak decay; one-meson-exchange: independent-particle shell model PACS: 21.80.+a, 13.75.Ev, 27.10.+h

The $\Lambda$-hypernuclei are mainly produced by the $\left(K^{-}, p^{-}\right)$and the $\left(p^{+}, K^{+}\right)$strong reactions, and disintegrate by the weak decay with the rate

$$
\Gamma_{W}=\Gamma_{M}+\Gamma_{N M},
$$

where $\Gamma_{M}$ is decay rate for the mesonic (M) decay $\Lambda \rightarrow \pi N$, and $\Gamma_{N M}$ is the rate for the nonmesonic (NM) decay, which can be induced either by one bound nucleon $(1 N)$, $\Gamma_{1}(\Lambda N \rightarrow n N)$, or by two bound nucleons $(2 N), \Gamma_{2}(\Lambda N N \rightarrow n N N)$, where $N=p, n$ i.e.,

$$
\Gamma_{N M}=\Gamma_{1}+\Gamma_{2} ; \quad \Gamma_{1}=\Gamma_{p}+\Gamma_{n} .
$$

In both cases the mass is changed by $176 \mathrm{MeV}$, and the strangeness by $\Delta \mathrm{S}=-1$, which makes the NM weak process to be the most radical modification of an elementary particle within the nuclear envarment, and offers the best opportunity to scrutinize the strangeness-changing force between hadrons. On the other hand, the hypernuclear physics leads to the extension of the radioactivity domain to three dimensions $(\mathrm{N}, \mathrm{Z}, \mathrm{S})$, which, because of the additional binding due to the $\Lambda$-hyperon, modifies the neutron drip line, and is even richer in elements than the ordinary $(\mathrm{N}, \mathrm{Z})$ domain. (For instance, while the one-neutron separation energy in ${ }^{20} \mathrm{C}$ is $1.01 \mathrm{MeV}$, it is $1.63 \mathrm{MeV}$ in ${ }_{\Lambda}^{21} \mathrm{C}$, and ${ }_{\Lambda}^{6} \mathrm{He}$ is bound while ${ }^{5} \mathrm{He}$ is unbound.) Because of this glue attribute of hypernuclei the $\Lambda N$ interaction is closely related to the inquiry on the existence of strange quark matter and its fragments, and strange stars (analogues of neutron stars), which makes the NM decay also relevant for astrophysics and cosmology.

The schematic representation of the two decay channels, when the pertinent dynamics is described by OME potentials, is shown in Fig. 1. This is the most frequently used 


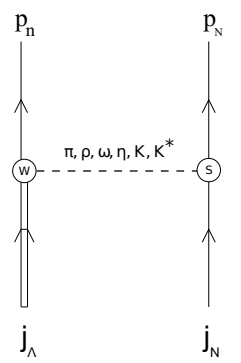

a)

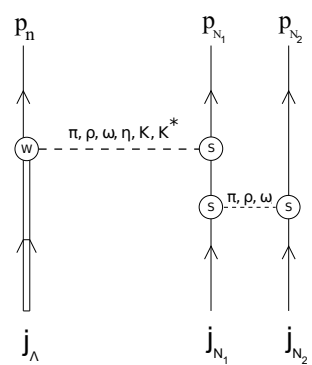

b)

FIGURE 1. Schematic representation of a) one-nucleon, and b) two-nucleon induced decays in $\Lambda$ hypernuclei when described by the interplay of weak $(W)$ and strong $(S)$ interactions through the exchanges of nonstrange-mesons $\pi, \rho, \omega$, and $\eta$, and strange-mesons $K$, and $K^{*}$.

model for handling the NM-decay, including usually the exchanges of nonstrangemesons $\pi, \rho, \omega$, and $\eta$, and strange-mesons $K$, and $K^{*}$. It is based on the original idea of Yukawa that the $N N$ interaction at long distance is due to the one-pion-exchange (OPE), the dominant role being played by the exchange of pion and kaon mesons.

Our present study involves the transition probability densities $S_{N}$ that can be obtained from the differentiation of the decay rates $\Gamma_{N}[1,2]$, and have been measured at the KEK [3], BNL [4], and FINUDA [5]. They are: 1) the single-proton spectra $S_{p}(E)$, and total neutron spectra $S_{n t}(E)=2 S_{p}(E)+S_{n}(E)$, as a function of corresponding one-nucleon kinetic energies $E_{N}$, and 2) two-particle-coincidence spectra, as a function of: i) the sum of kinetic energies $E_{n}+E_{N} \equiv E_{n N}, S_{n N}(E)$, ii) the opening angle $\theta_{n N}$, $S_{n N}(\cos \theta)$, and iii) the center of mass (c.m.) momentum $P_{n N}=\left|\mathbf{P}_{n N}=\mathbf{p}_{n}+\mathbf{p}_{N}\right|$ spectra $S_{n N}(P)$.

In Ref. [1] the calculated spectra are normalized to the experimental ones. For instance, for $S_{p}(E)$ the number of protons $\Delta \mathrm{N}_{p}^{e x p}\left(E_{i}\right)$, measured at energy $E_{i}$ within a fixed energy bin $\Delta E$, is compared with

$$
\Delta \mathrm{N}_{p}(E)=\frac{\mathrm{N}_{p}^{\exp }}{\Gamma_{p}} S_{p}(E) \Delta E,
$$

where $S_{p}(E)$, and $\Gamma_{p}=\int S_{p}(E) d E$ are evaluated theoretically, and $\mathrm{N}_{p}^{\exp }=$ $\sum_{i=1}^{m} \Delta \mathrm{N}_{p}^{\text {exp }}\left(E_{i}\right)$, being $m$ is the number of bins. Here, we are confronting $\Delta \mathrm{N}_{p}^{e x p}\left(E_{i}\right)$ straightforwardly with the corresponding calculated spectra, defined as

$$
\Delta \mathrm{N}_{p}\left(E_{p}\right)=\frac{\mathrm{N}_{W}^{\exp }}{\Gamma_{W}^{\exp }} S\left(E_{p}\right) \Delta E
$$

where $\mathrm{N}_{W}$ is the number of produced hypernuclei, and [6]

$$
\Gamma_{W}^{\exp }(A)=(0.990 \pm 0.094)+(0.018 \pm 0.010) A
$$

Unlike the equation (1), the relation (2) allows us to test not only the kinematics but also the dynamics. 

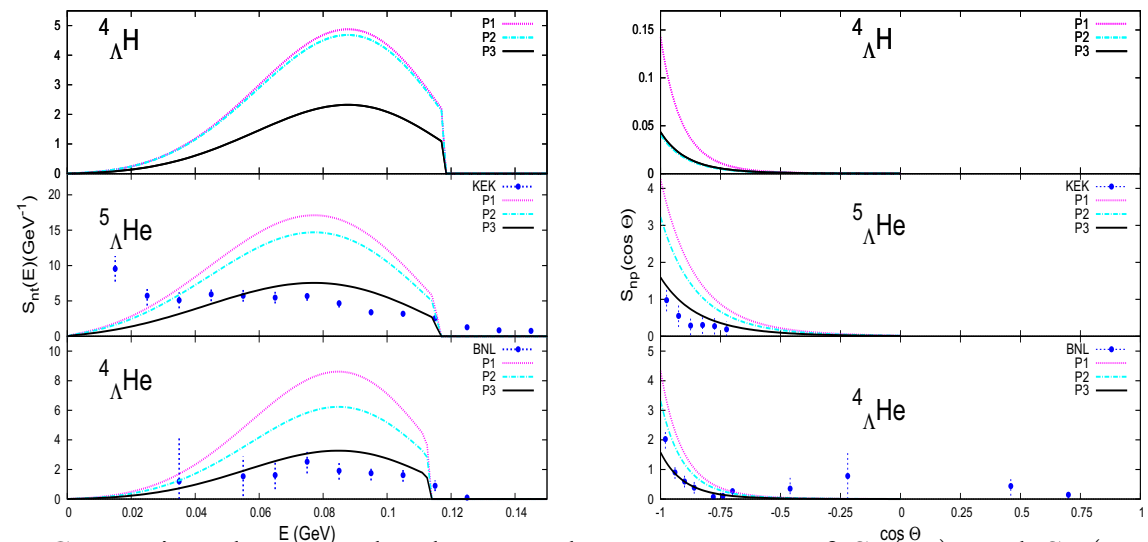

FIGURE 2. Comparison between the theory and measurements of $\left.S_{n t}^{\cos \theta} E_{n}\right)$, and $S_{n p}\left(\cos \theta_{n p}\right)$. The ${ }_{\Lambda}^{4} \mathrm{He}$ data are from BNL [4], and those for ${ }_{\Lambda}^{5} \mathrm{He}$ from KEK [3]. Predictions for ${ }_{\Lambda}^{4} \mathrm{H}$ are also displayed. The sharp cut of the spectra at high energy is due to the recoil.

Equivalently we compare the calculated spectrum $S_{p}(E)$ with the experimental one, defined as:

$$
S_{p}^{\exp }\left(E_{p}^{i}\right)=\left.\frac{\Gamma_{W}}{\mathrm{~N}_{W}} \frac{\Delta \mathrm{N}_{p}\left(E_{p}^{i}\right)}{\Delta E_{p}}\right|_{B N L, F I N U D A}=\left.\frac{\Gamma_{N M}}{\mathrm{~N}_{N M}} \frac{\Delta \mathrm{N}_{p}\left(E_{p}^{i}\right)}{\Delta E_{p}}\right|_{K E K} .
$$

The Independent Particle Shell Model (IPSM) is used for the nuclear structure framework, while the Solely one-nucleon-induced $(1 N-\mathrm{NM})$ decay is considered, omitting Final State Interactions (FSIs), and the two-nucleon-induced ( $2 N-\mathrm{NM})$ decay. For testing the dynamics we use three different OME potentials:

P1) The full pseudoscalar $(\pi, K, \eta)$ and vector $\left(\rho, \omega, K^{*}\right)$ meson octets (PSVE), with the weak coupling constants, and dipole form-factor cutoffs $\Lambda_{M}$ from Refs. [7, 8],

P2) Only one- $(\pi+K)$ exchanges (PKE) are considered, with the same parametrization as in the previous case, i.e., with cutoffs $\Lambda_{\pi}=1.3 \mathrm{GeV}$ and $\Lambda_{K}=1.2 \mathrm{GeV}$ from [8], and

P3) The soft $\pi+K$ exchange (SPKE) potential with cutoffs $\Lambda_{\pi}=0.7 \mathrm{GeV}$ and $\Lambda_{K}=0.9 \mathrm{GeV}$ from [1].

In Fig. 2 is shown the comparison between the theory and measurements done at BNL [4], and KEK [3] of $S_{n t}\left(E_{n}\right)$, and $S_{n p}\left(\cos \theta_{n p}\right)$ spectra in $s$-shell hypernuclei ${ }_{\Lambda}^{4} \mathrm{He}$, and ${ }_{\Lambda}^{5} \mathrm{He}$. The predictions for and ${ }_{\Lambda}^{4} \mathrm{H}$ are also presented. Similarly, in Fig. 3 is done the comparison in $S_{p}\left(E_{p}\right)$ for ${ }_{\Lambda}^{5} \mathrm{He},{ }_{\Lambda}^{7} \mathrm{Li},{ }_{\Lambda}^{9} \mathrm{Be},{ }_{\Lambda}^{11} \mathrm{~B},{ }_{\Lambda}^{12} \mathrm{C},{ }_{\Lambda}^{13} \mathrm{C},{ }_{\Lambda}^{15} \mathrm{~N}$ and ${ }_{\Lambda}^{16} \mathrm{O}$, recently measured by FINUDA [5]. In the last figure are exhibited as well the Gaussian-function fits of each proton spectrum from $80 \mathrm{MeV}$ onwards, which have been assumed in Ref. [5] to correspond to pure $1 N-\mathrm{NM}$ induced decay. The consequences of this assumption in relation to the $2 N-\mathrm{NM}$ induced decay will be discussed elsewhere.

With the parametrization P1 the theory greatly overestimates the spectra $S_{n t}\left(E_{n}\right)$, $S_{n p}\left(\cos \theta_{n p}\right)$, and $S_{p}\left(E_{p}\right)$ for all hypernuclei considered here, and especially for ${ }_{\Lambda}^{7} \mathrm{Li}$. The discrepancy can not be resolved by simply opening a new $2 N-\mathrm{NM}$ decay channel induced by two nucleons, since this decay mode, is incapable of quenching the total transition straight. To solve the problem we have to change the parametrization within the 1N-NM channel, or/and consider the FSIs, which can engender the excess of particles observed at low energies by scattering the high energy particles. Here we explore the 

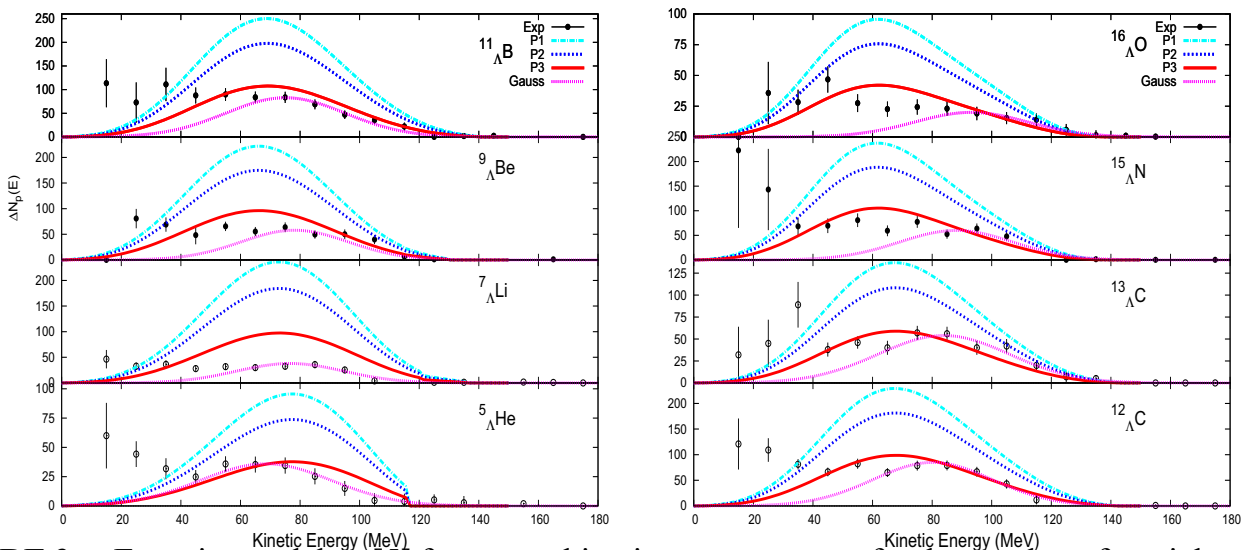

FIGURE 3. Experimental data [5] for proton kinetic energy spectra for the number of particles $\Delta \mathrm{N}_{p}\left(E_{p}\right)$ are compared with the IPSM results for three different OME potentials. Theoretical results have been evaluated from the relation (2) without considering experimental errors involved in $\mathrm{N}_{W}$ and $\Gamma_{W}$. Also are shown the Gaussian-function fits done in Ref. [5].

possibility of modifying the $1 \mathrm{~N}-\mathrm{NM}$ parametrization, assuming that the $2 \mathrm{~N}-\mathrm{NM}$ mode contributes dominantly at low energies. The agreement improves somewhat when the P1 model is substituted by the P2 one. However, spectra $S_{n t}\left(E_{n}\right), S_{n p}\left(\cos \theta_{n p}\right)$ in Fig. 2, and the high energy part of the proton spectra in Fig. 3 are reproduced in several hypernuclei $\left({ }_{\Lambda}^{5} \mathrm{He},{ }_{\Lambda}^{11} \mathrm{~B},{ }_{\Lambda}^{12} \mathrm{C},{ }_{\Lambda}^{13} \mathrm{C}\right.$, and $\left.{ }_{\Lambda}^{15} \mathrm{~N}\right)$ only with the parametrization P3. Yet, this is not the case of ${ }_{\Lambda}^{7} \mathrm{Li}$, and ${ }_{\Lambda}^{9} \mathrm{Be}$ where discrepancies continue to be significant.

In summary, the theory reproduces correctly the shapes of all spectra at medium and high energies $\left(E_{p} \gtrsim 40 \mathrm{MeV}\right)$, but it overestimates to a great extent their magnitudes, when is used the full OME model PSVE with standard parametrization. The agreement is much improved when is employed the SPKE model, i.e., the same model which reproduces satisfactorily the NM decays rates $\Gamma_{p}$ and $\Gamma_{n}$ of the s-shell hypernuclei [1].

\section{ACKNOWLEDGMENTS}

This work was partly supported by the Argentinean agencies CONICET (PIP 0377) and FONCYT (PICT-2010-2680), and by the Brazilian agencies FAPESP and CNPq.

\section{REFERENCES}

1. E. Bauer, A.P. Galeão, M. Hussein, F. Krmpotić, and J.D. Parker, Phys. Lett. B 674, 103 (2009).

2. F. Krmpotić, A. P. Galeão, and M.S. Hussein, AIP Conf. Proc. 1245, 51 (2010).

3. S. Okada, et al. , Phys. Lett. B 597 (2004) 249, B.H. Kang, et al. , Phys. Rev. Lett. 96, 025203 (2006), M.J. Kim, et al. , Phys. Lett. B641, 28 (2006).

4. J. D. Parker, et al. , Phys. Rev. C 76 (2007) 035501.

5. M. Agnello, et al., Phys. Lett. B685 (2010) 247.

6. M. Agnello et al. , Phys. Lett. B 681 (2009) 139.

7. J. F. Dubach, G. B. Feldman, B. R. Holstein, L. de la Torre, Ann. Phys. (N.Y.) 249 (1996) 146.

8. A. Parreño, A. Ramos, and C. Bennhold, Phys. Rev. C 56, 339 (1997). 
Copyright of AIP Conference Proceedings is the property of American Institute of Physics and its content may not be copied or emailed to multiple sites or posted to a listserv without the copyright holder's express written permission. However, users may print, download, or email articles for individual use. 